\title{
February 2018 Imaging Case of the Month
}

\author{
Michael B. Gotway, MD \\ Department of Radiology \\ Mayo Clinic Arizona \\ Scottsdale, AZ USA
}

Clinical History: An 18-year-old woman with a questionable history of asthma (one physician source claimed no clear history of asthma, whereas another source claimed severe asthma) presented to the emergency room with worsening shortness of breath and cough. The patient's past medical history was otherwise largely unremarkable. She did have complaints of recurrent rhinorrhea and allergies, for which sinus CT (Figure 1) had been performed.

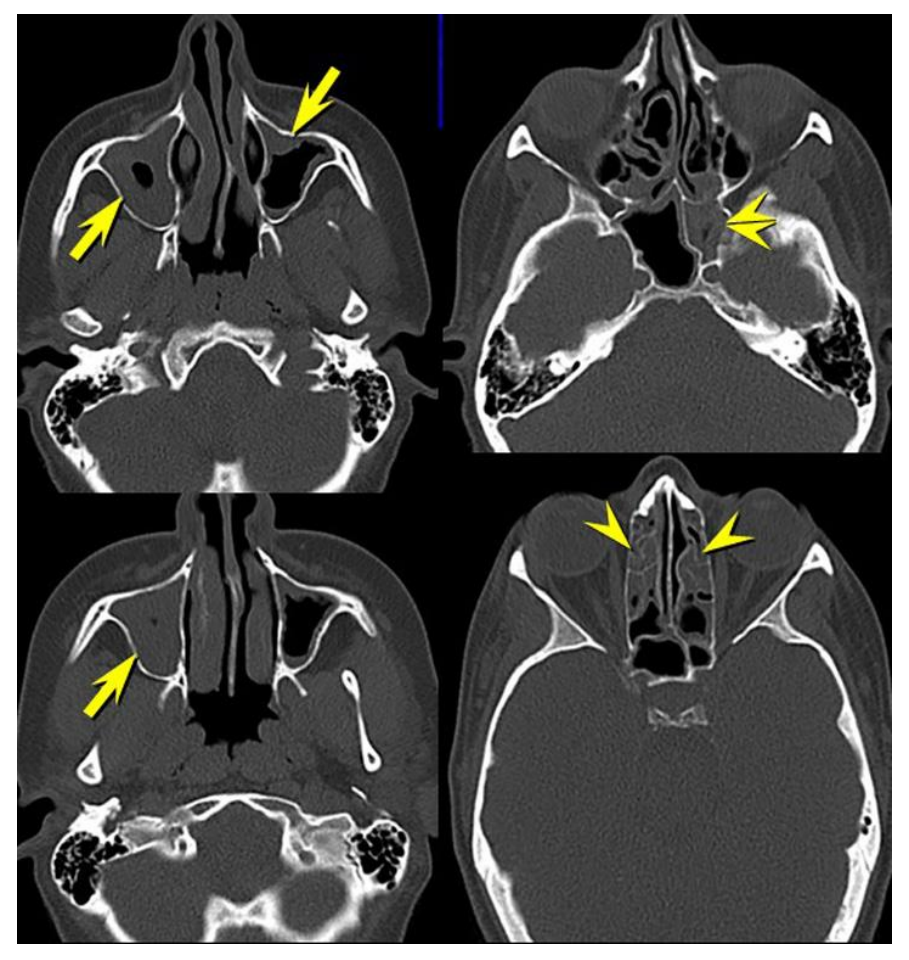

Figure 1. Unenhanced axial sinus CT shows multifocal sinus opacification (arrow = maxillary sinuses, arrowheads = ethmoid sinuses, double arrowhead= sphenoid sinus)

Physical examination was remarkable for coarse, right-greater-than-left basal rales and coarse breath sounds. The patient's oxygen saturation was $98 \%$ on room air. Her nasal septum appeared deviated. The patient's vital signs were within normal limits and she was afebrile.

Laboratory evaluation showed a normal complete blood count, electrolyte panel, and liver function tests. A digital frontal chest image (Figure 2) obtained at presentation is shown, with a comparison chest radiograph from 5 months earlier also shown. 


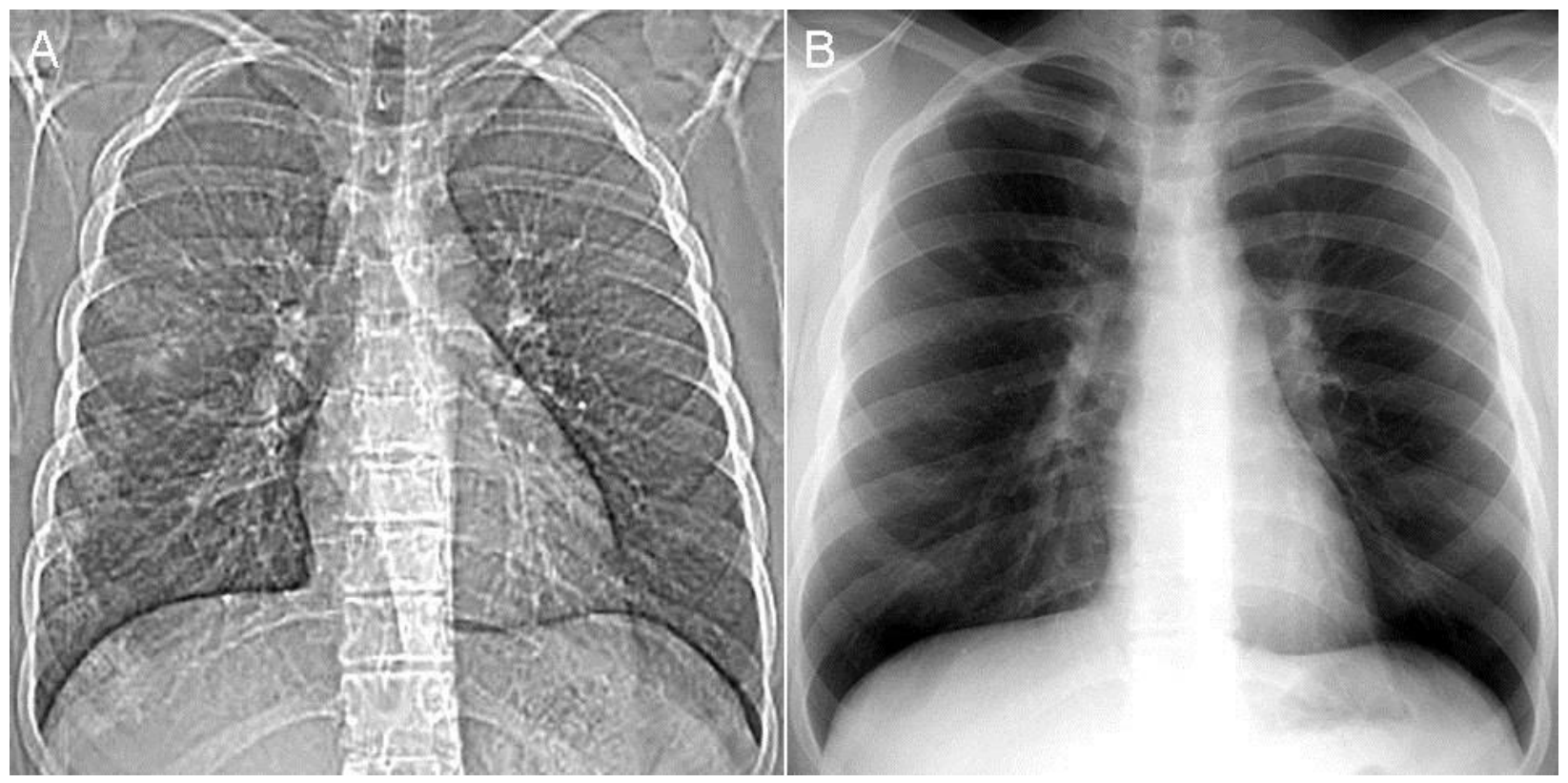

Figure 2. A: Digital frontal chest image. B: Chest radiograph from 5 months earlier.

Which of the following represents the most accurate assessment of the frontal chest imaging findings?

1. Chest frontal imaging shows basilar fibrosis

2. Chest frontal imaging shows mediastinal and peribronchial lymphadenopathy

3. Chest frontal imaging shows multiple, bilateral small nodules

4. Chest frontal imaging shows normal findings

5. Chest frontal imaging shows patchy nodular opacities in the right lung 


\section{Correct! \\ 5. Chest frontal imaging shows patchy nodular opacities in the right lung}

The frontal chest imaging shows faintly nodular opacity primarily in the peripheral right lung (Figure 3).

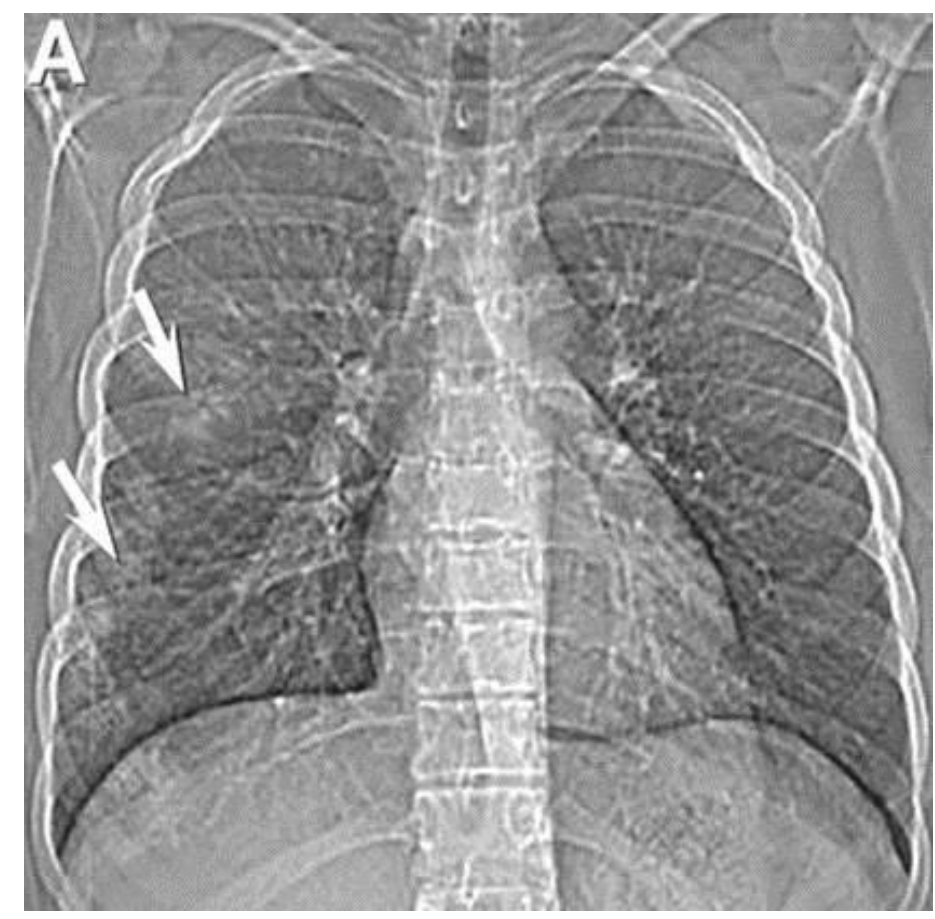

Figure 3. Frontal chest imaging shows poorly defined nodular opacity (arrows) primarily in the peripheral right mid and lower lung.

The lung volumes are normal and no clear abnormalities are seen in the left lung. No pleural abnormality is evident. There is no clear evidence of peribronchial or mediastinal lymph node enlargement.

The patient was treated in the emergency room with broad-spectrum antibiotics and prednisone, with equivocal improvement. While she initially felt better, she re-presented to the emergency room several weeks later with similar complaints, and was again treated with a different class of broad-spectrum antibiotics.

Which of the following represents the most appropriate management step for the evaluation of this patient?

1. Check titers for possible fungal infection, and again treat presumptively with broad-spectrum antibiotics for presumed pneumonia

2. Obtain ${ }^{18}$ FDG-PET scan

3. Obtain pulmonary function tests

4. Obtain surgical lung biopsy

5. Obtain thoracic MRI 


\section{Correct! \\ 3. Obtain pulmonary function tests}

Among the choices listed, obtaining pulmonary function testing is most appropriate. Repeat chest imaging would also be appropriate, but thoracic MR would not be a good choice among the available chest imaging modalities, given that the patient's primary imaging finding was a pulmonary abnormality. ${ }^{18} \mathrm{FDG}-\mathrm{PET}$ scan is premature at this point. While ${ }^{18}$ FDG-PET scan often proves useful for the investigation of focal pulmonary abnormalities, which this patient appears to have, usually ${ }^{18} \mathrm{FDG}-\mathrm{PET}$ scanning is most efficacious when focal, indeterminate pulmonary opacities are present and there is a consideration for either bronchogenic malignancy or secondary malignancy affecting the thorax. Given the lack of such suspicion at this point, and the patient's young age, which makes bronchogenic malignancy very unlikely, ${ }^{18} \mathrm{FDG}$-PET probably would not provide management altering data. For example, if the ${ }^{18} \mathrm{FDG}-\mathrm{PET}$ scan showed uptake within the right base, the differential diagnosis would still primarily center on infection, whereas negative results would not explain the patient's presentation and would be discordant with the recent chest imaging findings. Surgical lung biopsy is needlessly invasive at this point. Checking for fungal infection is certainly appropriate and should be performed, but a third course of presumptive broad-spectrum antibiotics, when two recent antibiotic courses were unsuccessful, would probably not benefit this patient.

The patient was seen by pulmonary medicine and started on bronchodilator inhalers, without much benefit. She saw another pulmonary medicine physician who started a prolonged course of prednisone, after which the patient reported feeling better. Several months later after her corticosteroids were tapered and withdrawn, she had with was thought to be an upper respiratory tract infection, after which her pulmonary medicine physician performed pulmonary function testing, which yielded the diagnosis of asthma. She also saw an allergist about this time who noted "petechial-like" lesions on her legs. Less than a month later, she was diagnosed with pneumonia and treated with azithromycin. Testing during this time showed positive IgM and IgA for Bordetella pertussis.

Which of the following represents the most appropriate management step for the evaluation of this patient?

1. Obtain ${ }^{18} \mathrm{FDG}$-PET scan

2. Obtain surgical lung biopsy

3. Obtain thoracic MRI

4. Perform bronchoscopy

5. Perform thoracic CT 


\section{Correct! \\ 5. Perform thoracic CT}

Given that some time has lapsed since the previous chest imaging showing an abnormality, and several medical interventions have been performed in the interim, it would be wise to repeat he chest imaging. This could be done with repeat chest radiography, but given the patient's already somewhat complicated course, thoracic CT would be preferable. Because the patient's clinical presentation and imaging findings have been primarily pulmonary, thoracic MR would be a less optimal approach to chest imaging than chest CT in this patient. As discussed previously, ${ }^{18}$ FDG-PET scan would likely not provide management-altering information for this patient at this point, and typically ${ }^{18} \mathrm{FDG}$-PET scanning is most useful when characterization of chest imaging findings with CT has been performed first. Surgical lung biopsy remains needlessly invasive at this point.

Unenhanced thoracic CT was performed (Figure 4).

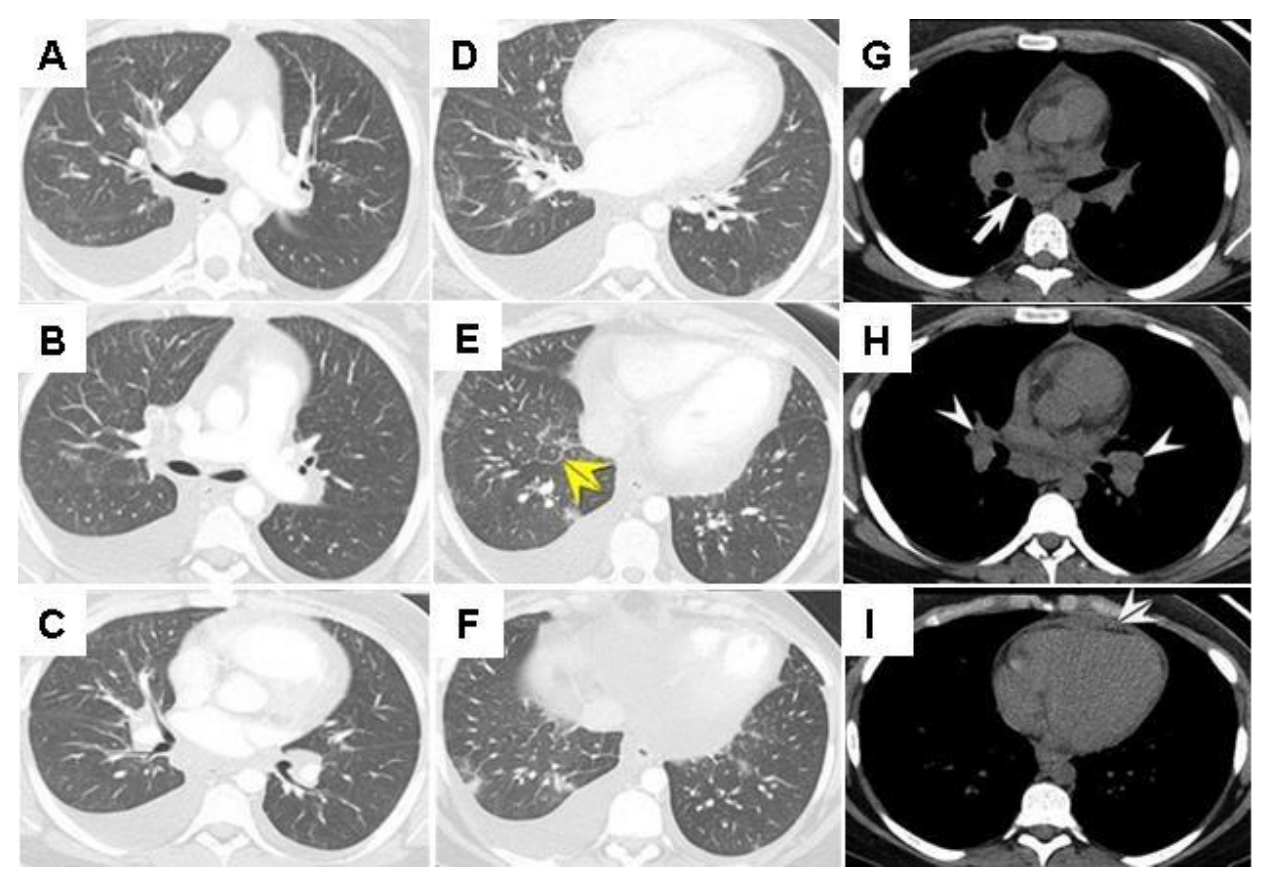

Figure 4. A-F: Representative images from thoracic CT in lung windows. G-I: Representative images in soft tissue windows.

Which of the following represents the most accurate assessment of the thoracic CT findings?

1. Thoracic CT shows bronchiectasis

2. Thoracic CT shows features suggesting fibrotic lung disease

3. Thoracic CT shows multifocal peripheral nodular opacities

4. Thoracic CT shows numerous small cavitary nodules

5. Thoracic CT shows numerous small perilymphatic nodules 


\section{Correct!}

\section{Thoracic CT shows multifocal peripheral nodular opacities}

The thoracic CT shows multifocal, bilateral areas of peripheral, and frankly subpleural, opacities. The opacities are nodular in some regions, but unassociated with features of fibrotic lung disease, such as traction bronchiectasis, coarse reticulation, architectural distortion, and honeycombing. Small perilymphatic nodules are not present and the pulmonary opacities are not cavitary. Mild symmetric peribronchial and mediastinal lymph node enlargement is present. The soft tissue windows show mild subcarinal (arrow) and symmetric bilateral (arrowheads) peribronchial lymph node enlargement. A small pericardial effusion (double arrowheads) is present.

A complete blood count showed a mildly elevated white blood cell count at $11.2 \times 10^{9} / \mathrm{L}$ (normal, $4-10 \times 10^{9} / \mathrm{L}$ ), but with $20 \%$ eosinophilia at $12 \times 10^{9} / \mathrm{L}$ (normal, $<0.45 \times 10^{9} /$ ). Serum chemistries and measures of renal function were within normal limits. Bronchoscopy was recommended to the patient, but she refused. Treatment of community-acquired pneumonia, presumably at least in part related to pertussis, was continued, as was oral corticosteroid therapy and inhalers for her asthma, but the patient still complained of shortness of breath and cough. Repeat thoracic CT (Figure 5) was performed less than one month following the previous thoracic CT.

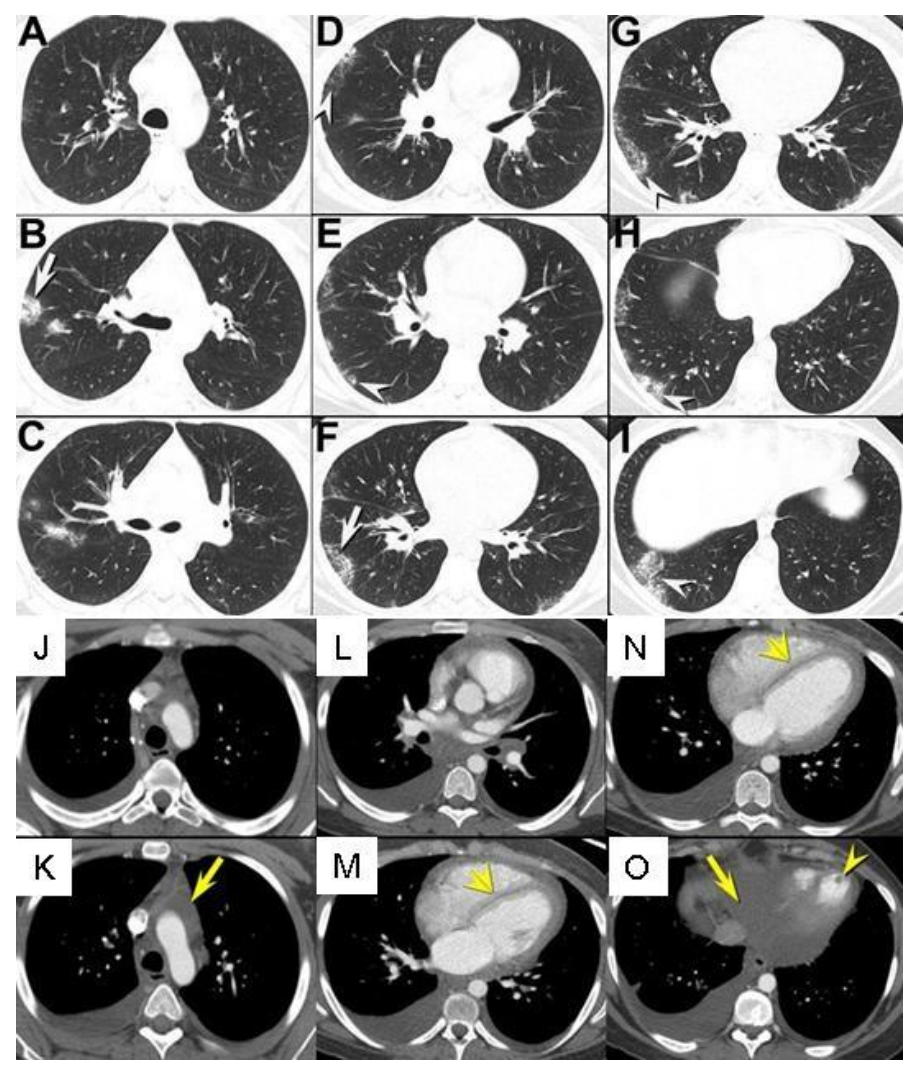

Figure 5. Repeat thoracic CT, at about 6 months following initial presentation, and less than one month following the first thoracic CT. A-I: Representative images displayed in lung windows. J-O: Representative images displayed in soft tissue windows. 
Which of the following represents the most accurate assessment of the thoracic CT findings?

1. The thoracic CT shows a new mediastinal mass

2. The thoracic CT shows interval improvement of the previously noted peripheral lung opacities

3. The thoracic CT shows interval worsening of the previously noted peripheral lung opacities

4. The thoracic CT shows new small nodules

5. The thoracic CT shows that the previously noted peripheral opacities are migrating 


\section{Correct! \\ 2. The thoracic CT shows interval improvement of the previously noted peripheral lung opacities}

The previously noted peripheral and subpleural opacities have significantly improved. No new nodules are present. However, the pericardial effusion persists, and new smooth interlobular septal thickening is present, associated with new right-greater-thanleft pleural effusions, and the myocardium shows an abnormal enhancement pattern. Furthermore, a small filling defect is seen within the apex of the left ventricle.

The patient was subsequently hospitalized and laboratory data showed the development of proteinuria, for which kidney biopsy was performed, showing acute tubular necrosis, but no glomerulonephritis. Echocardiography showed reduced left ventricular systolic function, left ventricular enlargement, and confirmed the present of left ventricular apical thrombus. Cardiac MRI (Figure 6) was performed and confirmed the presence of myocarditis as the cause of new left ventricular dysfunction and also demonstrated the left ventricular apical thrombus.

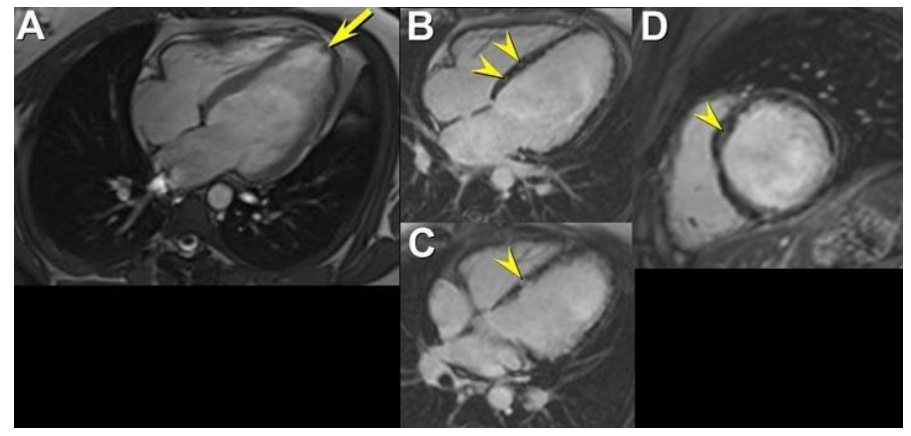

Figure 6. Cardiac MRI examination show left ventricular apical thrombus (arrow) within a dilated left ventricular cavity, and spotty areas of mid-wall delayed myocardial enhancement (arrowheads), the latter consistent with a non-ischemic cause of cardiomyopathy, particularly myocarditis. Left ventricular wall thickness is normal, arguing against hypertrophic cardiomyopathy and restrictive cardiomyopathy.

Serologic testing for cytoplasmic anti-neutrophil cytoplasmic antibody (c-ANCA) / proteinase-3 specificity was negative, but perinuclear anti-neutrophil cytoplasmic antibody ( $\mathrm{p}$-ANCA) / myeloperoxidase specificity testing was positive, and her serum $\lg$ E was elevated.

Given the foregoing information, which of the following represents the most likely diagnosis for this patient?

1. Chronic eosinophilic pneumonia

2. Coccidioidomycosis

3. Eosinophilic granulomatosis with polyangiitis (Churg-Strauss syndrome)

4. Organizing pneumonia

5. Undifferentiated connective tissue disorder 


\section{Correct! \\ 3. Eosinophilic granulomatosis with polyangiitis (Churg-Strauss syndrome)}

The patient has a number of features consistent with eosinophilic granulomatosis with polyangiitis, formerly referred to as Churg-Strauss syndrome. She has a history of recurrent rhinitis and CT scan evidence of paranasal sinusitis, possibly a history of nasal polyposis, and a clinical diagnosis of severe asthma. She has clear evidence of a non-ischemic cardiomyopathy with a cardiac MRI pattern suggestive of myocarditis, and elevated perinuclear anti-neutrophil cytoplasmic antibody ( $p-A N C A)$ with myeloperoxidase specificity. Importantly she also has evidence of peripheral eosinophilia (greater than 10\%) that appeared to be "unmasked" when her corticosteroid therapy was withdrawn, occurring in the context of fluctuating imaging opacities. While both chronic eosinophilic pneumonia and organizing pneumonia could result in the imaging opacities, and chronic eosinophilic pneumonia would be expected to produce the peripheral eosinophilia, neither chronic eosinophilic pneumonia nor organizing pneumonia would be expected to produce the myocarditis and left ventricular dysfunction noted at cardiac MRI. Some of this patient's presentation and imaging findings could fit with a connective tissue disorder, but the balance of the information available favors the diagnosis of eosinophilic granulomatosis with polyangiitis, aka, Churg-Strauss syndrome.

Diagnosis: Eosinophilic granulomatosis with polyangiitis (Churg-Strauss Syndrome)

\section{References}

1. Casta-er E, Alguersuari A, Andreu M, Gallardo X, Spinu C, Mata JM. Imaging findings in pulmonary vasculitis. Semin Ultrasound CT MR. 2012;33(6):567-79. [CrossRef] [PubMed]

2. Casta-er E, Alguersuari A, Gallardo X, Andreu M, Pallardó Y, Mata JM, Ramírez J. When to suspect pulmonary vasculitis: radiologic and clinical clues. Radiographics. 2010;30(1):33-53. [CrossRef] [PubMed]

3. Choi YH, Im JG, Han BK, Kim JH, Lee KY, Myoung NH. Thoracic manifestation of Churg-Strauss syndrome: radiologic and clinical findings. Chest. 2000;117(1):11724. [CrossRef] [PubMed]

4. Chung MP, Yi CA, Lee HY, Han J, Lee KS. Imaging of pulmonary vasculitis. Radiology. 2010;255(2):322-41. [CrossRef] [PubMed]

5. Feragalli B, Mantini C, Sperandeo M, Galluzzo M, Belcaro G, Tartaro A, Cotroneo $A R$. The lung in systemic vasculitis: radiological patterns and differential diagnosis. Br J Radiol. 2016;89(1061). [CrossRef] [PubMed]

6. Gross WL, Trabandt A, Reinhold-Keller E. Diagnosis and evaluation of vasculitis. Rheumatology (Oxford). 2000; 39(3):245-52. [CrossRef] [PubMed]

7. Semple D, Keogh J, Forni L, Venn R. Clinical review: Vasculitis on the intensive care unit--part 1: diagnosis. Crit Care. 2005 ;9(1):92-7. [CrossRef] [PubMed]

8. Semple D, Keogh J, Forni L, Venn R. Clinical review: Vasculitis on the intensive care unit -- part 2: treatment and prognosis. Crit Care. 2005; 9(2):193-197. [CrossRef] [PubMed] 